\title{
Interactive Exploration of Fuzzy RDF Knowledge Bases
}

\author{
Nikos Manolis and Yannis Tzitzikas \\ Institute of Computer Science, FORTH-ICS, Greece, and \\ Computer Science Department, University of Crete, Greece \\ \{manolisn,tzitzik\}@ics.forth.gr
}

\begin{abstract}
In several domains we have objects whose descriptions are accompanied by a degree expressing their strength. Such degrees can have various application specific semantics, such as relevance, precision, certainty, trust, etc. In this paper we consider Fuzzy RDF as the representation framework for such "weighted" descriptions and associations, and we propose a novel model for browsing and exploring such sources, which allows formulating complex queries gradually and through plain clicks. Specifically, and in order to exploit the fuzzy degrees, the model proposes interval-based transition markers. The advantage of the model is that it significantly increases the discrimination power of the interaction, without making it complex for the end user.
\end{abstract}

\section{Introduction}

There are many practical situations where the descriptions of objects are accompanied by a degree. These degrees can be provided by humans or be the result of automated tasks. For instance, in 19] they express the degree of a feature that is extracted from data, in [7] they express the certainty of membership of a document to an aspect (automatically retrieved from the answer set of a keyword query), in [17] the membership of an object to a cluster, in [8] the evaluation scores of products under hierarchically organized criteria, in [13] they express the frequency of symptoms in a disease. Furthermore in an open environment like the Web, we may have data of various degrees of credibility, as well data which are copies or modifications of other data. As a result, data of the same entity can be erroneous, out-of-date, or inconsistent/conflicting in different data sources. Therefore, even if the primary data are not fuzzy, the integrated data as produced by an information integration system (that contains tasks like web extraction) can be fuzzy. Synopsizing, such degrees can capture various application specific semantics, such as relevance, precision, certainty, trust, etc.

Users would like to browse and explore such sources without having to be aware of the terminology, contents or query language of the source. Furthermore, the fuzzy degrees should be exploitable, allowing the users to reach states which are characterized by conditions that involve degrees. Finally, it should be possible to offer adequate support for recall-oriented information needs, and support a 
gradual focus restriction process (i.e. interactive search). However there is not any exploration/browsing approach for such sources.

The contribution of this paper lies in introducing a model for exploring such sources. The merit of the proposed model is that it defines formally and precisely the state space of an interaction that (a) allows users to locate the objects of interest, or to get overviews, without having to be aware of the terminology nor the query language of the underlying source, and without reaching states with empty results, (b) exploits fuzzy degrees for enhancing the discrimination power of the interaction, (c) generalizes the main exploration/browsing approaches for plain RDF/S sources (also clarifying issues regarding schema and instance cyclic property paths), (d) is query language independent, and (e) is visualization independent. Finally it discusses issues that concern the available query languages.

To grasp the idea, Fig. 1 shows an example of a Fuzzy RDF KB where instance triples have fuzzy degrees. Properties are depicted by rectangles and the letters "d" and "r" denote the domain and the range of a property. Fat arrows denote subClassOf/subPropertyOf relationships, while dashed arrows denote instanceOf relationships. Note that various approaches have been proposed recently for annotating triples with a degree of truth and giving a semantics to such annotations, e.g. 16 18, and can be used as the representation framework. Among the various possible approaches for exploiting fuzzy degrees at the interaction level, we propose one based on intervals, leading to a simple and intuitive dialog. The idea is to analyze the count information of each transition marker to more than one counts each corresponding to the objects whose membership degrees fall into a specified interval. An instance of the proposed interaction is sketched at Fig. 2, The figure depicts only the part of the UI (usually the left bar) that shows the transition markers (it does not show the object set). Fig. 2(a) ignores fuzzy degrees. Fig. 2(b) shows how fuzzy degrees are exploited. For example, consider a transition marker " $\mathrm{z}(20)$ ". We can present it as "z $[\operatorname{low}(10), \operatorname{medium}(4), \operatorname{high}(6)]$ " where "low" may correspond to degrees $(0,0.3]$, "medium" to $(0.3,0.6]$ and "high" to $(0.6,1]$. So the user has now three clicks (i.e. three transitions) instead of one. Therefore the set of all possible foci is more, so the discrimination power of interaction increases. The increased discrimination power is useful especially in cases where the fuzzy degrees are derived by automatic methods. Since the results of such methods are vulnerable to errors and inaccuracies (i.e. the derived degrees may be lower or higher than those deserved), it would not be wise to exclude the descriptions having low degrees. Instead, it is better to make all of them available and let the user free to explore also the objects having low degrees if necessary. In brief the interaction leads to states whose extension corresponds to the answer of complex path expressions that involve interval-based conditions over the fuzzy degrees, e.g. queries of the form: "Japanese cars for sale which are driven by persons who work at FORTH and know a person who knows Bob", where each individual underlined part (value-based condition) is associated also with an interval-based condition over the fuzzy degrees.

The paper is organized as follows. Section 2 describes in brief related works. Section 3 introduces the least number of symbols and notations required for 


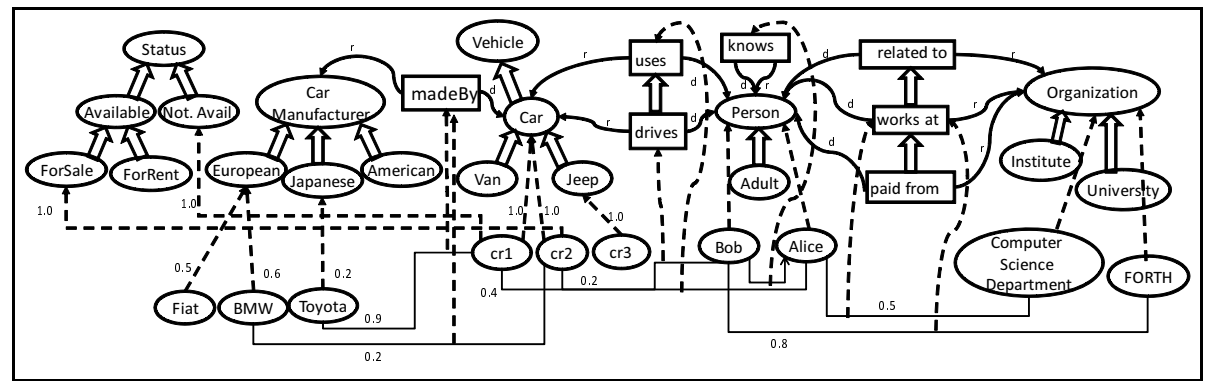

Fig. 1. A Fuzzy RDF KB

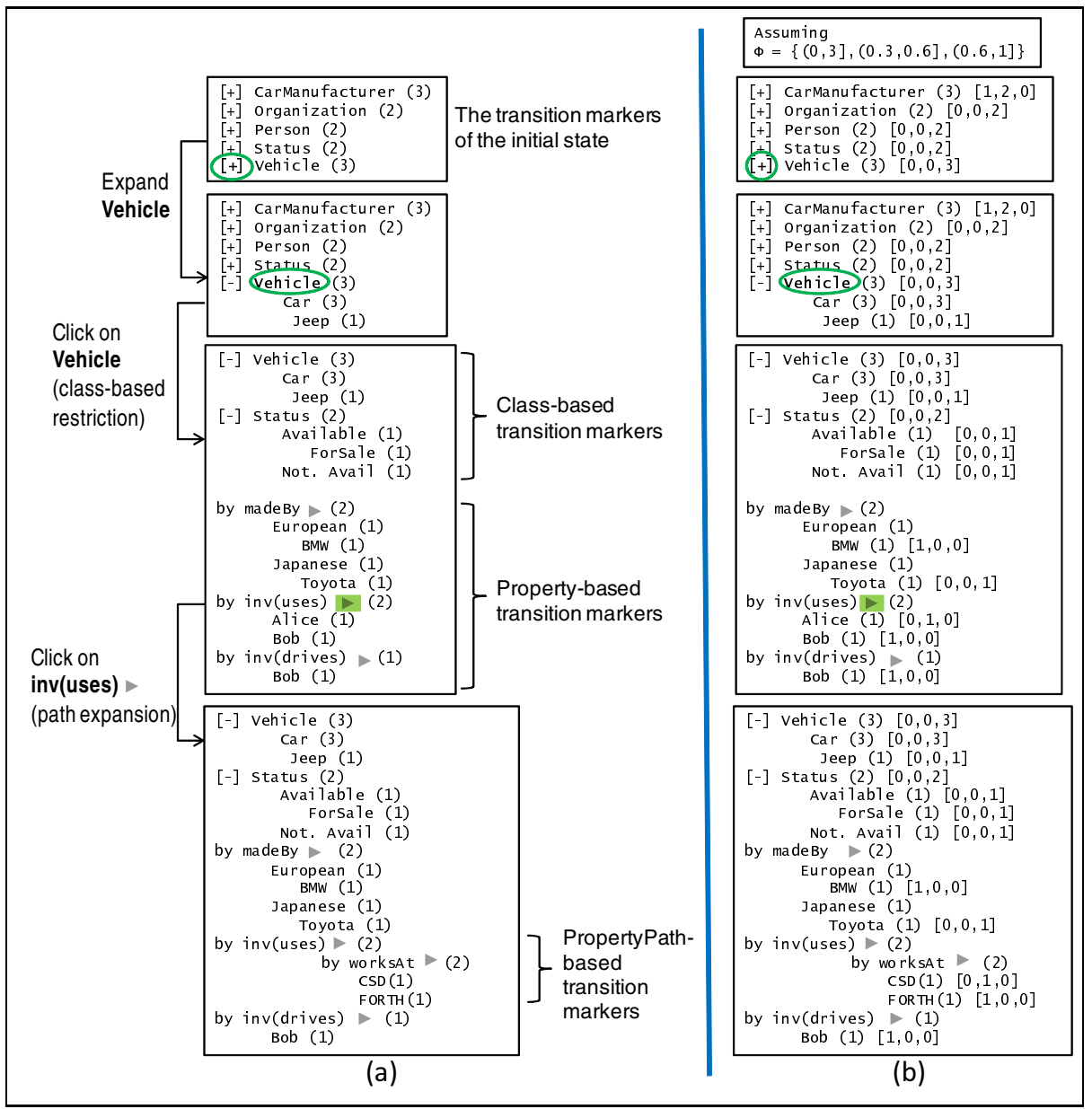

Fig. 2. Sketch of the GUI part for transition markers

(a): ignores fuzzy degrees. (b): count information based on intervals over the fuzzy degrees 
defining the interaction model. Section 4 introduces the model first for plain $\mathrm{RDF} / \mathrm{S}$ and then extends it for Fuzzy RDF. Section [5] discusses query language issues, and finally Section 6 concludes the paper.

\section{Related Work}

Some browsing approaches are applicable to simple structures (like attributevalue pairs), while others to complex information structures (e.g. OWL-based $\mathrm{KBs}$ ). Therefore one important aspect is how the underlying information is structured. There are several options, some of them follow: attribute-value pairs with flat values (e.g. name=Yannis), attribute-value pairs with hierarchically organized values (e.g. location=Crete), set-valued attributes (either flat or hierarchical) (e.g. accessories $=\{$ ABS, ESP $\}$ ), multi-entity (or object-oriented) (e.g. RDF, Linked Open Data), and relational databases. Furthermore, we could have fuzziness and we can consider this as an independent aspect (e.g. there are fuzzy extensions of the RDF model such as [1016]).

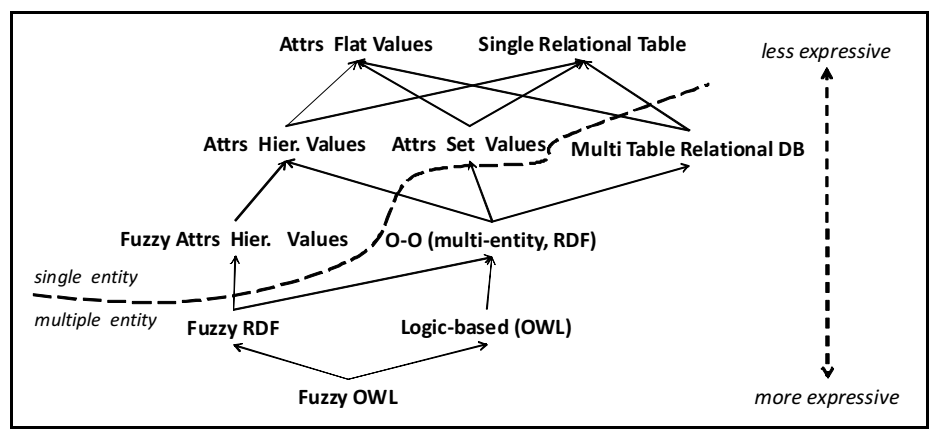

Fig. 3. Categories of Information Spaces

Fig. 3 shows the above categories organized hierarchically where an option $\mathrm{X}$ is a (direct or indirect) child of an option $\mathrm{Y}$ if whatever information can be expressed in $\mathrm{Y}$ can also be expressed in $\mathrm{X}$. The value of this diagram is that it depicts the fact that if a browsing approach is appropriate for an option $\mathrm{X}$, then certainly it is appropriate for all options which are parents of X. For instance, a browsing approach appropriate for Fuzzy RDF is also appropriate for plain RDF, as well as for sources formed by attributes with fuzzy and hierarchically organized values. Just indicatively, the following table lists some browsing approaches grouped according to their applicability. In this paper we focus on Fuzzy RDF and we are interested in generic approaches (not requiring special configuration), that exploit schema information (if available). In contrast, 6. deals with fuzzy annotations of documents w.r.t. a particular ontology using a view-based approach where the browsable elements (views) are predefined by specialists. 


\begin{tabular}{|l|l|}
\hline Information Space & System \\
\hline \hline Attrs with flat values & Elastic Lists [14] \\
\hline Attrs with hierarchical values & Flamenco [20], Mitos WSE [12] \\
\hline Object-Oriented (e.g. RDF) & GRQL [2], BrowseRdf[11, Ontogator [9], VisiNav 4 \\
\hline FRDF (Fuzzy RDF) & Fuzzy view-based Semantic Search 6] \\
\hline OWL & Odalisque [ \\
\hline
\end{tabular}

\section{Fuzzy RDF}

Consider a set of RDF triples $K$ and let $\mathcal{C}(K)$ be its closure. We shall denote with $C$ the set of classes, with $\operatorname{Pr}$ the set of properties, with $\leq_{c l}$ the subclassOf relation between classes, and with $\leq_{p r}$ the subpropertyOf relation between properties. The instances of a class $c \in C$ are $\operatorname{inst}(c)=\{o \mid(o$, type, $c) \in \mathcal{C}(K)\}$, while the instances of a property $p \in \operatorname{Pr}$ are $\operatorname{inst}(p)=\left\{\left(o, p, o^{\prime}\right) \mid\left(o, p, o^{\prime}\right) \in \mathcal{C}(K)\right\}$. Now we introduce some notations for Fuzzy RDF. Each instance triple $t$ (either class or property instance triple) is accompanied by a fuzzy degree, which we shall denote by directdegree $(t)$. We can now define the degree of $t$, denoted by degree $(t)$, based on the semantics of RDF, and the axioms of Fuzzy Set Theory1. Specifically,

degree $(o$, type,$c)=\max \left\{\right.$ directdegree $\left(o\right.$, type,$\left.\left.c^{\prime}\right) \mid c^{\prime} \leq_{c l} c\right\}$

degree $\left(o, p, o^{\prime}\right)=\max \left\{\right.$ directdegree $\left.\left(o, p^{\prime}, o^{\prime}\right) \mid p^{\prime} \leq_{p r} p\right\}$.

Let $\Phi=\left\{\varphi_{1}, \ldots \varphi_{m}\right\}$ be a set of intervals in $[0,1]$. We define:

$\operatorname{inst}(c, \varphi)=\{o \in \operatorname{inst}(c) \mid$ degree $(o$, type,$c) \in \varphi\}$

$\operatorname{inst}(p, \varphi)=\left\{\left(o, p, o^{\prime}\right) \in \operatorname{inst}(p) \mid \operatorname{degree}\left(o, p, o^{\prime}\right) \in \varphi\right\}$.

\section{The Interaction Model}

The interaction is modeled by a state space. Each state has an extension and a number of transitions leading to other states. Each transition is signified by a transition marker accompanied by a number showing the size of the extension of the targeting state (we will refer to this with count information). Such view abstracts from the various visualization approaches; in general each state has one or more visualization modes for its extension as well as its transition markers.

\subsection{The Interaction Model for Plain RDF/S}

The objective is to define a precise and concise model capturing the essentials of RDF browsing approaches, which later will be extended to capture Fuzzy RDF.

Consider that we are in the context of one RDF/S KB with a single namespace with classes $C$ and properties $\operatorname{Pr}$. If $s$ denotes a state we shall use $s . e$ to denote its extension. Let's start from the initial state(s). Let $s_{0}$ denote an artificial initial state. We can assume that $s_{0} . e=U R I \cup L I T$, i.e. its extension contains every URI and literal of the KB. Alternatively, the extension of the initial state can

${ }^{1}$ We will adopt Zadeh's theory and consequently we shall use min/max, however one could also adopt alternative definitions for the operators $\otimes, \bigoplus$ (e.g. as done in [16]). 
be the result of a keyword query, or a set of resources provided by an external access method. Given a state we shall show how to compute the transitions that are available to that state. From $s_{0}$ the user can move to states corresponding to the maximal classes and properties, i.e. to one state for each maximal $_{\leq_{c l}}(C)$ and each maximal $_{\leq_{p r}}(P r)$. Specifically, each $c \in$ maximal $_{\leq_{c l}}(C)$ (resp. $p \in$ maximal $\left._{\leq_{p r}}(P r)\right)$ yields a state with extension inst(c) (resp. inst $\left.(p)\right)$.

We will define formally the transitions based on the notion of restriction and join. To this end we introduce some auxiliary definitions. We shall use $p^{-1}$ to denote the inverse direction of a property $p$, e.g. if $(d, p, r) \in \operatorname{Pr}$ then $p^{-1}=(r, i n v(p), d)$, and let $\operatorname{Pr}^{-1}$ denote the inverse properties of all properties in $\operatorname{Pr}$. If $E$ is a set of resources, $p$ is a property in $\operatorname{Pr}$ or $\operatorname{Pr}^{-1}, v$ is a resource or literal, vset is a set of resources or literals, and $c$ is a class, we define the following notations for restricting the set $E$ :

$$
\begin{aligned}
\operatorname{Restrict}(E, p: v) & =\{e \in E \mid(e, p, v) \in \operatorname{inst}(p)\} \\
\operatorname{Restrict}(E, p: \text { vset }) & =\left\{e \in E \mid \exists v^{\prime} \in \text { vset and }\left(e, p, v^{\prime}\right) \in \operatorname{inst}(p)\right\} \\
\operatorname{Restrict}(E, c) & =\{e \in E \mid e \in \operatorname{inst}(c)\}
\end{aligned}
$$

Now we define a notation for joining values, i.e. for computing values which are linked with the elements of $E$ :

$$
\operatorname{Joins}(E, p)=\{v \mid \exists e \in E \text { and }(e, p, v) \in \operatorname{inst}(p)\}
$$

We can now define precisely transitions and transition markers. Suppose we are in a state $s$ with extension s.e.

Class-based browsing. The classes that can be used as class-based transition markers, denoted by $T M_{c l}(E)$, are defined by:

$$
T M_{c l}(E)=\{c \in C \mid \operatorname{Restrict}(E, c) \neq \emptyset\}
$$

If the user clicks on a $c \in T M_{c l}(s . e)$, then the extension of the targeting state $s^{\prime}$ is defined as $s^{\prime} . e=$ Restrict $(s . e, c)$, and its count information is $s^{\prime}$.count $=\left|s^{\prime} . e\right|$. For example, suppose the user selects the class Vehicle. The user can then view its instances and follow one of the following class-based transition markers: Vehicle, Car, Jeep, Status, Available, ForSale, Not.Available. Notice that ForRent and Van are not included because their extension (and thus their intersection with the current extension) is empty.

The elements of $T M_{c l}$ (s.e) can be hierarchically organized (based on the subclass relationships among them). Specifically the layout (e.g. the indentation in a text-based layout) of the transition markers can be based on the relationships of the reflexive and transitive reduction of the restriction of $\leq_{c l}$ on $T M_{c l}$ (s.e) (i.e. on $R^{\text {refl,trans }}\left(\leq_{c l} \mid T M_{c l}(\right.$ s.e $\left.)\right)$ ). In our case, we can get what is shown in Fig. 4 (a). Furthermore based on the relationship between the extensions s.e and $s^{\prime} . e$ a transition (or transition marker) can be characterized as a zoom-in/out/side transition.

Let's now focus on property-based browsing. Suppose the user has focused on Car, and the extension of this state is $\{\operatorname{cr} 1, \operatorname{cr} 2, \operatorname{cr} 3\}$. He can further 
restrict the extension also through the properties. Roughly each property whose domain or range is the class Car, or a superclass of Car (in general any property that used in the resources in s.e), can be considered as a facet of the instances of Car. For example, consider a property madeBy whose domain is the class Car and suppose its range was the String Literal class. In that case the firm names of the current extension can be used as transition markers. Now suppose that the range of the property madeBy is not literal, but the class CarManufacturer (as shown in figure). In this case, the firms (URIs in this case) of the current extension can again be used as transition markers, as shown in Fig. 4(b). Notice that Fiat is not shown as it is not related to the current focus (i.e. to cr1, cr2 and $\operatorname{cr} 3)_{2}^{2}$. Formally, the properties (in their defined or inverse direction) that can be used for deriving transition markers are defined by:

$$
\operatorname{Props}(s)=\left\{p \in \operatorname{Pr} \cup \operatorname{Pr}^{-1} \mid \operatorname{Joins}(\text { s.e, } p) \neq \emptyset\right\}
$$

For each $p \in \operatorname{Props}(s)$, the corresponding transition markers are defined by Joins $(s . e, p)$, and if the user clicks on a value $v$ in Joins $(s . e, p)$, then the extension of the new state is $s^{\prime} . e=\operatorname{Restrict}(s . e, p: v)$.

(a)

Vehicle(3)
Car(3)
Jeep(1)
Status (2)
Available(1)
ForSale(1)
Not. Avail(1)

(b)

by madeBy (2) by madeB $\operatorname{BMW}(1)$

$\operatorname{Toyota}(1)$

(c)

BMW (1)

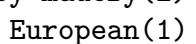

Japanese (1)

Toyota (1)

(d)

by inv(uses) (2)

Alice (1)

Bob (1)

by inv(drives) (1)

Bob (1) (e)

by inv(uses) (2)

by worksAt (2)

CSD (1)

FORTH (1)

Fig. 4. Examples of transition markers

Furthermore, the transition markers of a property $p \in \operatorname{Props}(s)$, i.e. the set Joins $(s . e, p)$, can be categorized based on their classes. In our example, the firms can be categorized through the subclasses of the class CarManufacturer. These classes can be shown as intermediate nodes of the hierarchy that lead to particular car firms, as shown in Fig. 4 (c). These classes can be computed easily, they are actually given by $T M_{c l}(\operatorname{Joins}(s . e, p))$. Furthermore, these values can be used as complex transition markers, i.e. as shortcuts allowing the user to select a set of values with disjunctive interpretation (e.g. he clicks on Japanese instead of clicking to every Japanese firm). Specifically, suppose the user clicks on such a value $v c$. The extension of the target state $s^{\prime}$ will be:

$$
s^{\prime} . e=\operatorname{Restrict}(s . e, p: \operatorname{Restrict}(\operatorname{Joins}(s . e, p), v c))
$$

\footnotetext{
${ }^{2}$ Since cr3 does not participate to a madeBy property, an alternative approach is to add an artificial value, like NonApplicable/Uknown, whose count would be equal to 1 , for informing the user that one element of his focus has not value wrt madeBy.
} 
Returning to our example, and while the user has focused on cars, apart from madeBy, the user can follow transitions based on the properties inv(drives) and inv(uses), as shown in Fig. 4(d). In addition, the elements of Props ( $s$ ) can be hierarchically organized based on the subProperty relationships among them.

We should be able to extend the above for property paths of length greater than one. This is needed for restricting the extension through the values of complex attributes (e.g. addresses that may be represented as blank nodes) or through the relationships (direct or indirect) with other resources. For example, one may want to restrict the set of cars so that only cars which are used by persons working for CSD (Computer Science Department) are shown. In that case we would like transition markers of the form shown in Fig. 4(e). It should also be possible the successive "application" of the same property. For example, the user may want to focus to all friends of the friends of Bob, or all friends of Bob at distance less than 5. Let's now define precisely, this property path-based browsing (expansion and cascading restriction). Let $p_{1}, \ldots, p_{k}$ be a sequence of properties. We call this sequence successive in $s$ if Joins (Joins $\left(\ldots\left(\right.\right.$ Joins $\left.\left.\left(s . e, p_{1}\right), p_{2}\right) \ldots p_{k}\right)$ $\neq \emptyset$. Obviously such a sequence does not lead to empty results, and can be used to restrict the current focus. Let $M_{1}, \ldots M_{k}$ denote the corresponding set of transition markers at each point of the path. Assuming $M_{0}=$ s.e, the transition markers for all $i$ such that $1 \leq i \leq k$, are defined as:

$$
M_{i}=\operatorname{Joins}\left(M_{i-1}, p_{i}\right)
$$

What is left to show is how selections on such paths restrict the current focus. Suppose the user selects a value $v_{k}$ from $M_{k}$. This will restrict the set of transitions markers in the following order $M_{k}, \ldots, M_{1}$ and finally it will restrict the extension of $s$. Let $M_{k}^{\prime}, \ldots M_{1}^{\prime}$ be the restricted set of transitions markers. They are defined as follows: $M_{k}^{\prime}=\left\{v_{k}\right\}$, while for each $1 \leq i<k$ we have:

$$
M_{i}^{\prime}=\operatorname{Restrict}\left(M_{i}, p_{i+1}: M_{i+1}^{\prime}\right)
$$

for instance, $M_{1}^{\prime}=\operatorname{Restrict}\left(M_{1}, p_{2}: M_{2}^{\prime}\right)$. Finally, the extension of the new state $s^{\prime}$ is defined as $s^{\prime} . e=\operatorname{Restrict}\left(s . e, p_{1}: M_{1}^{\prime}\right)$. Equivalently, we can consider that $M_{0}^{\prime}$ corresponds to $s^{\prime} . e$ and in that case Eq. 5 holds also for $i=0$.

For example, consider an ontology containing a path of the form: Car--hasFirm-->Firm--ofCountry-->Country and three cars cr1, cr2, cr3, the first being BMW, the second VW, the third Renault. The first two firms come from Germany the last from France. Suppose the user is on Cars, and expands the path hasFirm. of Country. If he selects Germany, then the previous list will become BMW, VW (so Renault will be excluded) and the original focus will be restricted to $\operatorname{cr} 1$ and $\mathrm{cr} 2$. It follows that path clicks require disjunctive interpretation of the matched values in the intermediate steps.

The above can be applied also for successive applications of the same property, e.g. inv(drives).knows ${ }^{2}$. paidFrom is a property path that can be used to restrict cars to those cars whose drivers know some persons who in turn know some persons who are paid from a particular organization. 
Entity Type Switch. So far we have described methods to restrict the current extension. Apart from the current extension we can move to other objects. At the simplest case, from one specific resource we move to one resource which is directly or indirectly connected to that. Now suppose that the current focus is a set of resources (e.g. cars). Again we can move to one or more resources which are directly or indirectly connected (to all, or at least one) of the resources of the current focus. For example, while viewing a set of cars we can move to (and focus on) the list of their firms (in this way we interpret disjunctively the elements associated with every object of the focus). To capture this requirement it is enough to allow users to move to a state whose extension is the current set of transition markers. For example, consider a user who starts from the class Persons, and then restricts his focus to those persons who workAt FORTH. Subsequently he restricts his focus through the property drives, specifically he restricts his focus to European. At that point he asks to change the entity type to Cars. This means that the entity type of the extension of the new state should be Cars, and the extension of the new state will contain European cars which are driven by persons working at FORTH. The property drives (actually its inverse direction), is now a possible facet of the current focus (and a condition is already active, based on the session of the user). Furthermore, the user can proceed and restrict his focus (European cars which are driven by persons working at FORTH) to those which are ForSale, and so on.

\subsection{The Interaction Model for Fuzzy RDF}

The general idea is that each transition of the model is now analyzed into $|\Phi|$ transitions, one for each $\varphi \in \Phi$, and each one is signified by its count (therefore now we will have $|\Phi|$ instead of 1 counts). To define these transitions we extend the previous definitions so that each of them takes an interval as additional parameter. Specifically, each Restrict takes as input an additional parameter $\varphi$, and the same for Joins, i.e.:

$$
\begin{aligned}
\operatorname{Restrict}(E, c, \varphi) & =\{e \in E \mid e \in \operatorname{inst}(c, \varphi)\} \\
\operatorname{Restrict}(E, p: v, \varphi) & =\{e \in E \mid(e, p, v) \in \operatorname{inst}(p, \varphi)\} \\
\operatorname{Restrict}(E, p: v \operatorname{vset}, \varphi) & =\left\{e \in E \mid \exists v^{\prime} \in \operatorname{vset} \text { and }\left(e, p, v^{\prime}\right) \in \operatorname{inst}(p, \varphi)\right\} \\
\operatorname{Joins}(E, p, \varphi) & =\{v \mid \exists e \in E \text { and }(e, p, v) \in \operatorname{inst}(p, \varphi)\}
\end{aligned}
$$

Regarding class-based transitions, it follows that for each $\mathrm{tm}$ in $T M_{c l}($ s.e) we now have one $T M_{c l}($ s.e, $\varphi)$ for each $\varphi \in \Phi$, where:

$T M_{c l}(s . e, \varphi)=\{c \in C \mid \operatorname{Restrict}(s . e, c, \varphi) \neq \emptyset\}$, and if the user clicks on a $c \in T M_{c l}(s . e, \varphi)$, then $s^{\prime} . e=\operatorname{Restrict}(s . e, c, \varphi)$.

Regarding property-based transitions, for each $p \in \operatorname{Props}(s)$, the corresponding transition markers in plain $\mathrm{RDF}$ were defined by Joins $(s . e, p)$. Now, each element in Joins $(s . e, p)$ is analyzed to one Joins $(s . e, p, \varphi)$ for each $\varphi \in \Phi$. If the user clicks on a value $v$ in $\operatorname{Joins}(s . e, p, \varphi)$, then $s^{\prime} . e=\operatorname{Restrict}(s . e, p: v, \varphi)$. Regarding presentation, we do not show intervals, instead we show the corresponding count information. For example, for each $e \in E=\bigcup_{\varphi \in \Phi} T M_{c l}(s . e, \varphi)$ we show $e$ once and its counts for each $\varphi \in \Phi$. Analogously for properties. 
Let's now focus on property paths. For example consider two property instances $p i_{1}$ and $p i_{2}$ that form a path (e.g. (cr2, inv (uses) , Bob, 0.2) and (Bob, worksAt, CSD , 0.8)), each associated with a fuzzy degree $d_{1}$ and $d_{2}$ respectively. The degree of path $p i_{1} \cdot p i_{2}$ is $\min \left(d_{1}, d_{2}\right)$, in our case 0.2 , since each path actually corresponds to a conjunction. This means that if the user's focus is cars and he wants to restrict it through the organization of the users of the cars, then the path $p i_{1} \cdot p i_{2}$ will be taken into account for computing the count of the transition marker CSD whose interval encloses the degree $\min \left(d_{1}, d_{2}\right)$. To define this precisely, we first introduce some notations. Let $p p=p_{1}, \ldots, p_{k}$ be a property path. An instance path of $p p$ is a sequence of the form $i p=\left(v_{0}, p_{1}, v_{1}\right) \cdot \ldots \cdot\left(v_{k-1}, p_{k}, v_{k}\right)$ where for all $1 \leq i \leq k:\left(v_{i-1}, p_{i}, v_{i}\right) \in \mathcal{C}(K)$. The degree of an instance path $i p$ is defined as the minimum degree of its edges (property instance triples). The degree of a path from o to $o^{\prime}$ over $p p$, denoted as degree $\left(o, p p, o^{\prime}\right)$, is the maximum degree of all instance paths of $p p$ between these two objects. We can now define joins and restrictions based on fuzzy paths:

$$
\begin{aligned}
\operatorname{Joins}(E, p p, \varphi) & =\left\{v_{k} \mid \exists e \in E \operatorname{such} \text { that degree }\left(e, p p, v_{k}\right) \in \varphi\right\} \\
\operatorname{Restrict}\left(E, p p: v_{k}, \varphi\right) & =\left\{e \in E \mid \operatorname{degree}\left(e, p p, v_{k}\right) \in \varphi\right\}
\end{aligned}
$$

Now we will analyze the algorithmic aspect of the above (since the previous two definitions were declarative). Consider a property path $p p=p_{1} \cdot \ldots \cdot p_{k}$. The transition markers at each stage are defined as before, i.e. $M_{i}=\operatorname{Joins}\left(M_{i-1}, p_{i}\right)$. For each individual element $e \in$ s.e we define the set of transition markers of level $i$ (where $1 \leq i \leq k$ ) which are associated with it, as:

$$
\operatorname{ETM}_{i}(e)=\left\{m_{i} \in M_{i} \mid \exists s \in \operatorname{ETM}_{i-1}(e) \text { and } \quad\left(s, p_{i}, m_{i}\right) \in \operatorname{inst}\left(p_{i}\right)\right\}
$$

assuming that $\operatorname{ETM}_{0}(e)=\{e\}$.

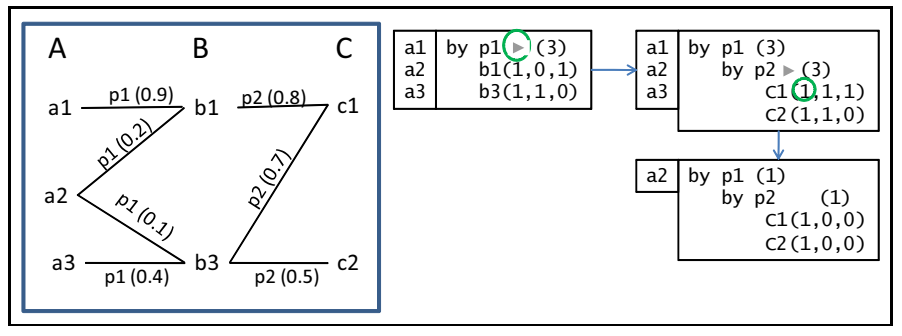

Fig. 5. Interaction over fuzzy paths

For example consider the case shown at Fig. 5. Let $A$ be the extension of the current state $\left(M_{0}=A=\right.$ s.e). For a path consisting only of one property $p_{1}$ we have that $M_{1}=\operatorname{Joins}\left(M_{0}, p_{1}\right)=\{b 1, b 3\}$, while for $p_{1} \cdot p_{2}$ we have $M_{2}=\operatorname{Joins}\left(M_{1}, p_{2}\right)=\{c 1, c 2\}$. Now, for each element $e \in$ s.e we have the following sets of transition markers of level $i$ : 
level 0: $\operatorname{ETM}_{0}(a 1)=\{a 1\}$ level 1: $\operatorname{ETM}_{1}(a 1)=\{b 1\}$ level 2: $\operatorname{ETM}_{2}(a 1)=\{c 1\}$

$$
\begin{array}{ll}
\operatorname{ETM}_{0}(a 2)=\{a 2\} & \operatorname{ETM}_{0}(a 3)=\{a 3\} \\
\operatorname{ETM}_{1}(a 2)=\{b 1, b 3\} & \operatorname{ETM}_{1}(a 3)=\{b 3\} \\
\operatorname{ETM}_{2}(a 2)=\{c 1, c 2\} & \operatorname{ETM}_{2}(a 3)=\{c 1, c 2\}
\end{array}
$$

In addition, for each element $e \in$ s.e and transition marker $m_{i} \in \operatorname{ETM}_{i}(e)$, we introduce a value denoted by $\operatorname{Deg}\left(e, m_{i}\right)$, which is actually the degree of a path from $e$ to $m_{i}$ over $p p$ (note that if $p p$ is empty then we assume $\operatorname{Deg}(e, e)=1$ ). This value can be computed gradually (i.e. as the path gets expanded) as follows:

$$
\operatorname{Deg}\left(e, m_{i}\right)=\max _{m_{i-1} \in E T M_{i-1}(e)}\left\{\min \left(\operatorname{degree}\left(m_{i-1}, p_{i}, m_{i}\right), \operatorname{Deg}\left(e, m_{i-1}\right)\right)\right\}
$$

In our example we have: $\operatorname{Deg}(a 2, b 1)=\max _{a \in E T M_{0}(a 2)}\left\{\min \left(\right.\right.$ degree $\left(a, p_{1}, b 1\right)$, $\operatorname{Deg}(a 2, a))\}=\min \left(\operatorname{degree}\left(a 2, p_{1}, b 1\right), \operatorname{Deg}(a 2, a 2)\right)=0.2$. Analogously, $\operatorname{Deg}(a 2$, $b 3)=0.1$. Now, the degree of $a 2$ to the transition marker $c 1$ is computed as: $\operatorname{Deg}(a 2, c 1)=\max _{b \in E T M_{1}(a 2)}\left\{\min \left(\operatorname{degree}\left(b, p_{2}, c 1\right), \operatorname{Deg}(a 2, b)\right)\right\}=$ $\max \left\{\min \left(\right.\right.$ degree $\left.\left(b 1, p_{2}, c 1\right), \operatorname{Deg}(a 2, b 1)\right), \min \left(\right.$ degree $\left.\left.\left(b 3, p_{2}, c 1\right), \operatorname{Deg}(a 2, b 3)\right)\right\}$ $=\max \{\min (0.8,0.2), \min (0.7,0.1)\}=\max \{0.2,0.1\}=0.2$. Analogously, $\operatorname{Deg}(a 1, c 1)=0.8$ and $\operatorname{Deg}(a 3, c 1)=0.4$.

Finally, the count for each $m_{i}$ of $M_{i}$ that corresponds to $\varphi$ is given by:

$$
\operatorname{count}\left(m_{i}, \varphi\right)=\mid\left\{e \in \text { s.e } \mid \operatorname{Deg}\left(e, m_{i}\right) \in \varphi\right\} \mid
$$

e.g. at Fig. [5 and for $\varphi=(0,0.3]$, we have $\operatorname{count}(c 1, \varphi)=1$. By clicking on the count $\operatorname{count}\left(m_{i}, \varphi\right)$ the extension of the current state is restricted as follows $s^{\prime} . e=\left\{e \in\right.$ s.e $\left.\mid \operatorname{Deg}\left(e, m_{i}\right) \in \varphi\right\}$.

\subsection{Path Expansion and Cycles}

Here, we examine how the interaction model for Fuzzy RDF behaves in case we have cycles at schema and instance level. At schema level, a property sequence may have the same starting and ending class forming a cycle (cyclic properties can be considered as a special case where the length of the sequence is 1 ). In the context of the proposed interaction model, when we have a cyclic schema path (e.g. $p p=$ inv (uses). worksAt. owns as it is shown in Fig. 6) we may reach transitions markers which are also elements of the initial focus s.e, e.g. a transition marker $m_{i}$ such that $m_{i} \in$ s.e, and we may have to compute its $\operatorname{Deg}\left(m_{i}, m_{i}\right)$. Consider the property path $p_{1} \cdot p_{2} \cdot p_{3} \cdot p_{4}$ where $p_{1}=i n v(u s e s)$, $p_{2}=$ knows, $p_{3}=$ works At, $p_{4}=$ owns. If $c r 1$ belongs to s.e, then $\operatorname{ETM}_{0}(\mathrm{cr} 1)=$ $\{c r 1\}, \operatorname{ETM}_{1}(\mathrm{cr} 1)=\{$ Alice $\}, \operatorname{ETM}_{2}(\mathrm{cr} 1)=\{B o b\}, \operatorname{ETM}_{3}(\mathrm{cr} 1)=\{C S D\}$ and $\operatorname{ETM}_{4}(\operatorname{cr} 1)=\{c r 1\}$. To compute the appropriate count information of the transition marker $\operatorname{cr} 1$, it is enough to compute $\operatorname{Deg}(\operatorname{cr} 1, \operatorname{cr} 1)$ according to Eq. 9] as the path $p p=p_{1} \cdot p_{2} \cdot p_{3} \cdot p_{4}$ is not empty. The point is that the proposed model can handle cycles at schema level without requiring any further configuration.

Now consider a user who wants to restrict the initial focus set, being a set of persons, through other persons connected with them through the property knows at depth $m$. For this reason the user expands the property knows $m$ times and then selects a person. However, at some point we may want to stop 


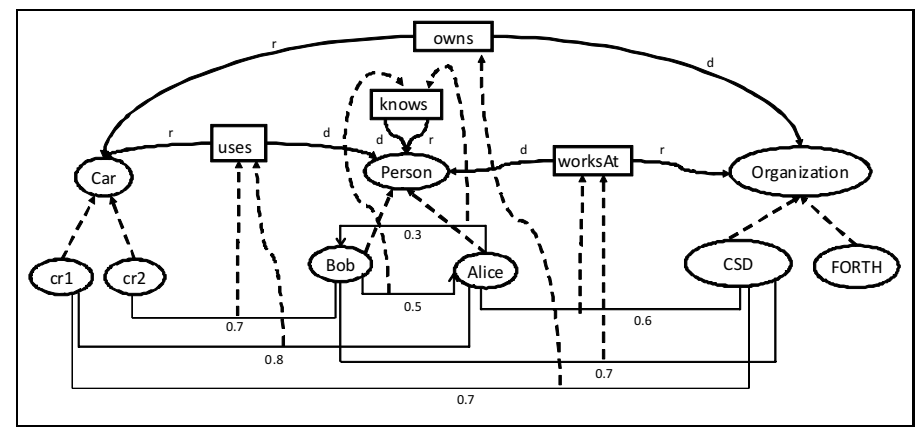

Fig. 6. A Fuzzy RDF graph with cycles at schema and instance level

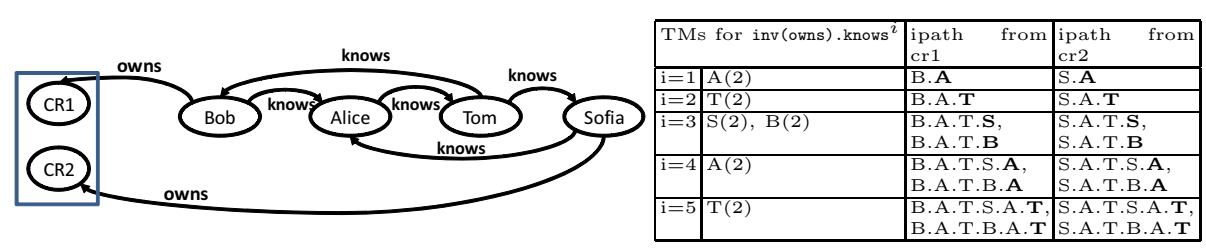

Fig. 7. Instance cycles example

suggesting path expansions, in order to avoid prompting to the user the same set of transition markers (which may periodically restrict the initial focus in the same way). For example, in Fig. 7 we can see that when the property knows is expanded over 3 times, the transition markers and their count info is periodically being repeated 3 . We propose adopting the following policy: stop path expansion when each object of the s.e has been made accessible (i.e. restrictable) through all transition markers that are possible. Below we explain how we can compute the max number of expansion steps. Let $\Gamma=(N, R)$ be a directed graph. A path from $n_{1}$ to $n_{k+1}$, is a sequence of edges of the form $\left(n_{1}, n_{2}\right) \ldots\left(n_{k}, n_{k+1}\right)$ where $\left(n_{i}, n_{i+1}\right) \in R$, and $i \neq j$ implies that $n_{i} \neq n_{j}$. The length of such a path is $k$, and we shall write $n_{1} \stackrel{k}{\rightsquigarrow} n_{k+1}$ to denote that there exists a path of length $k$ from $n_{1}$ to $n_{k+1}$. We shall also write $n \stackrel{*}{\rightsquigarrow} n^{\prime}$ to denote that exists one or more paths from $n$ to $n^{\prime}$. Now we define the distance from $n$ to $n^{\prime}$ as the length of the shortest path from $n$ to $n^{\prime}$, i.e. $\operatorname{Dist}\left(n \rightarrow n^{\prime}\right)=\min \left\{k \mid n \stackrel{k}{\rightsquigarrow} n^{\prime}\right\}$. Given two subsets $A$ and $B$ of $N$ (i.e. $A, B \subseteq N$ ), we define the distance from $A$ to $B$ as the maximum distance between any pair of nodes form these sets, i.e. $\operatorname{Dist}(A, B)=\max \{\operatorname{Dist}(a \rightarrow b) \mid a \in A, b \in B\}$.

Returning to our problem $N$ is the set of all nodes of the RDF graph. For a property $p \in \operatorname{Pr}$ we can define the edges $R_{p}=\{(a, b) \mid(a, p, b) \in \mathcal{C}(K)\}$. We can

${ }^{3}$ Only the first letter of a name is shown and paths over knows are depicted as sequences of such letters. 
now define the reachable nodes from a node $n$ (through $p$ property instances) as Reachable $_{p}(n)=\left\{n^{\prime} \mid n \stackrel{*}{{ }^{*}} n^{\prime}\right\}$, where the meaning of the subscript $p$ is that paths are formed from elements of $R_{p}$. Being at a state $s$, the maximum number of path expansion steps (for property $p$ ) that is required are:

$$
\operatorname{MaxExpansionSteps}(s, p)=\operatorname{Dist}\left(\text { s.e }, \bigcup_{n \in s . e} \operatorname{Reachable}_{p}(n)\right)
$$

With this number of steps it is guaranteed that each object of s.e has been made accessible (restrictable) through all tms (transition markers) which are possible. The proof is trivial: the path starting from an object $o$ with length bigger than MaxExpansionSteps $(s, p)$ will not encounter a tm that has not already been reached.

Now consider path expansions over different properties (i.e. inv (owns) . knows . knows). In such cases we would like to to identify the maximum expansion steps for each $p$ that is used in the expansion, or the maximum expansion steps in general. Let pset be a set of properties (i.e. pset $\subseteq P r)$. We can define the edges by considering all properties in pset, i.e. $R_{\text {pset }}=\{(a, b) \mid p \in$ pset, $(a, p, b) \in \mathcal{C}(K)\}$. Now we can define Reachable Rset $(n)=\left\{n^{\prime} \mid n{ }^{*}{ }_{\text {pset }}^{*} n^{\prime}\right\}$, where the subscript pset means that paths consist of edges in $R_{\text {pset }}$. The set Reachable pset $(n)$ is the set of all tms through which $n$ is accessible. Therefore the tms of all objects in s.e, which are accessible through paths using property instances in pset, are given by $\bigcup_{n \in s . e}$ Reachable $_{p s e t}(n)$. Being at a state $s$, the maximum number of path expansion steps (using properties from pset) that is required is:

$$
\text { MaxExpansionSteps }(s, \text { pset })=\operatorname{Dist}\left(\text { s.e }, \bigcup_{n \in s . e} \operatorname{Reachable}_{\text {pset }}(n)\right)
$$

\section{Query Language Issues}

We have defined the interaction model using only extensions (not intentions), since the expression of the intention depends on the Query Language (QL), or the abstraction of the QL that one adopts. However the underlying information source may be accessible through a QL. Table1 shows the notation we have used for defining RDF browsing, and their expression in SPARQL. In this description we consider that the extension of the current state is stored in a class with name ns: temp 4 . Furthermore we assume that the closure of the KB is stored. However, we should note that Virtuoso [3], supports an extended SPARQL version with subclassOf and subproperty inference at query level. This means that triples entailed by subclass or subproperty statements are not physically stored, but they are added to the result set during query answering 5. This means that the SPARQL expressions of Table 1 would not require another change.

\footnotetext{
${ }^{4}$ Instead of ns:temp we could have a set of SPARQL graph patterns. For reasons of space, we do not describe the query construction method.

${ }^{5}$ Similar in spirit with the online approach [5] to query the Web of Linked Data by traversing RDF links during run-time.
} 
Table 1. SPARQL-expression of Notations for RDF Browsing

\begin{tabular}{|c|c|}
\hline Notation & Expression in SPARQL \\
\hline $\begin{array}{l}\text { Restrict }(E, p \quad: \quad \text { vset }), \\
\text { vset }=\left\{v_{1}, \ldots, v_{k}\right\}\end{array}$ & $\begin{aligned} \text { select } & ? \mathrm{x} \text { where }\{? \mathrm{x} \text { rdf:type ns:temp; ns:p ?V. } \\
& \left.\text { Filter }\left(? \mathrm{~V}=\mathrm{ns}: \mathrm{v}_{-} 1|| \ldots|| \mathrm{V}=\mathrm{ns}: \mathrm{v}_{-} \mathrm{k}\right)\right\}\end{aligned}$ \\
\hline Restrict $(E, c)$ & select ?x where $\{$ ?x rdf:type ns:temp; rdf:type ns:c.\} \\
\hline $\begin{array}{l}\text { Joins }(E, p), \text { where } E= \\
\left\{e_{1}, \ldots, e_{k}\right\}\end{array}$ & $\begin{array}{l}\text { select Distinct ?v where }\{? \mathrm{x} \text { ns:p ?v. } \\
\left.\text { Filter }\left(? \mathrm{x}=\mathrm{ns}: \mathrm{e}_{-} 1|| \ldots|| ? \mathrm{x}=\mathrm{ns}: \mathrm{e}_{-} \mathrm{k}\right)\right\}\end{array}$ \\
\hline$T M_{c l}(s . e)$ and counts & $\begin{array}{l}\text { select Distinct ?c count(*) where\{?x rdf:type ?c; rdf:type ns:temp.\} } \\
\text { group by ?c }\end{array}$ \\
\hline $\operatorname{Props}(s)$ & $\begin{array}{r}\text { select Distinct ?p where }\{\text { ? } \mathrm{x} \text { rdf: type ns:temp; ?p ?v. }\} \\
\text { UNION \{?m rdf:type ns:temp. ?n ?p ?m. }\}\}\end{array}$ \\
\hline Joins $(s . e, p)$ and counts & $\begin{array}{l}\text { select Distinct ?v count }(*) \text { where }\{\text { ? } \text { rdf:type ns:temp; ns:p ?v.\} } \\
\text { groupby ?v }\end{array}$ \\
\hline
\end{tabular}

Regarding QLs for Fuzzy RDF, there is not any standardized (or widely adopted) extension of SPARQL. Some recently proposed deductive systems, e.g. [16 15, support fuzzy answering over unions of conjunctive queries, by computing the closure of a Fuzzy RDF graph (i.e. degree(o,type,c) is computed as we have defined it, however degree $\left(o, p, o^{\prime}\right)$ is not directly supported), storing it into a relational DB, and then using internally SQL queries. For instance, [16] uses MonetDB with the following schema: type (subject, object, degree), subclassOf (subject, object, degree), subpropertyOf (subject, object, degree), and a table $\operatorname{prop}_{i}$ (subject, object, degree) for every distinct property $p_{i}$. Table 2 shows directly the SQL queries that are needed by our interaction model for Fuzzy RDF (again $E$ could also be defined through another query).

Table 2. SQL-expression of Notations for Fuzzy RDF Browsing

\begin{tabular}{|c|c|}
\hline Notation & Expression in SQL \\
\hline Restrict $(E, c, \varphi)$ & $\begin{array}{l}\text { select subject from type } \\
\text { where object='c' and subject in } \mathrm{E} \\
\text { and degree>phi.low and degree }<=\text { phi.up }\end{array}$ \\
\hline Restrict $(E, p:$ vset,$\varphi)$ & $\begin{array}{l}\text { select subject from } \mathrm{p} \\
\text { where object in VSET and subject in } \mathrm{E} \\
\text { and degree }>\text { phi. Iow and degree }<=\text { phi.up }\end{array}$ \\
\hline Joins $\left(E, p_{i}\right)$ & select object from $p_{-} i$ where subject in $\mathrm{E}$ \\
\hline$T M_{c l}(s, \varphi)$ and counts & $\begin{array}{l}\text { select object, sum(case when degree>phi.lower } \\
\quad \text { and degree <= phi.upper then } 1 \text { else } 0 \text { end), } \\
\text { from type } \\
\text { where subject in s.e group by object }\end{array}$ \\
\hline $\operatorname{ETM}\left(e, M_{i}\right.$, Prev $)$ & $\begin{array}{l}\text { select object from } \mathrm{P}_{-} i \\
\text { where subject in Prev and object in } \mathrm{M}_{-} i\end{array}$ \\
\hline $\begin{array}{l}\operatorname{Deg}_{M I N}\left(e, s u b j, m_{i}, d\right) \\
\text { where } \operatorname{subj} \in \operatorname{ETM} M_{i-1}(e) \\
\text { and } d=\operatorname{Deg}(e, s u b j)\end{array}$ & $\begin{array}{l}\text { select } \\
\text { case when degree > d then d else degree end as DEG_MIN } \\
\text { from } p \text { where subject='subj' and object='m_i', }\end{array}$ \\
\hline
\end{tabular}

Regarding property paths, at each step we can compute $M_{i}=\operatorname{Joins}\left(M_{i-1}, p_{i}\right)$ with a single SQL query (as shown in Table 2). The difference of fuzzy paths vs non fuzzy paths, is that for moving from a stage $i$ of the path to a stage $i+1$, we have for each $e \in$ s.e to keep (a) $\operatorname{ETM}_{i}(e)$, and (b) $\operatorname{Deg}\left(e, m_{i}\right)$ for each $m_{i} \in E T M_{i}(e) \subseteq M_{i}$. To compute $\operatorname{ETM}_{i}(e)$ we can use a query of the form $\operatorname{ETM}\left(e, M_{i}\right.$, Prev) (shown in Table 2) where Prev $=E T M_{i-1}(e)$. To compute $\operatorname{Deg}\left(e, m_{i}\right)$ we can use a query of the form $\operatorname{Deg}_{M I N}\left(e, s u b j, m_{i}, d\right)$ for every $s u b j \in E T M_{i-1}(e)$ (and accordingly $d=\operatorname{Deg}(e, s u b j)$ ) and then get the max. 


\section{Conclusion}

We proposed a session-based interaction model for exploring Fuzzy RDF KBs in a simple and intuitive manner. To exploit fuzzy degrees the model supports interval-based transition markers and this (exponentially) increases the discrimination power of the interaction. Roughly, for each condition of a (formulated on the fly) query, we have $|\Phi|$ refinements of that condition. This means that for states corresponding to queries with $k$ conditions we now have $|\Phi|^{k}$ more states. This increase does not affect the friendliness of the interaction since only states leading to non-empty results are given. We also analyzed the query language requirements for realizing this model on top of the query layer. We do not include experimental measurements, since this is not the focus of this paper (and for reasons of space), however we should mention that to compute the class-based transition markers, and their fuzzy counts, for a dataset with $10^{7}$ instances can take up to 3 secs for the case of $|\Phi|=5$ in MonetDB 6 . Directions for further research regard ranking methods for the transition markers. For instance, fuzzy degrees can be exploited for clustering transition markers, or for ranking them through more refined methods than those proposed for plain RDF (e.g. 11]).

\section{References}

1. Allard, P., Ferré, S.: Dynamic Taxonomies for the Semantic Web. In: Procs. of the 19th Intern. Conf. on Database and Expert Systems Application, DEXA (2008)

2. Athanasis, N., Christophides, V., Kotzinos, D.: Generating On the Fly Queries for the Semantic Web: The ICS-FORTH Graphical RQL Interface (GRQL). In: McIlraith, S.A., Plexousakis, D., van Harmelen, F. (eds.) ISWC 2004. LNCS, vol. 3298, pp. 486-501. Springer, Heidelberg (2004)

3. Erling, O., Mikhailov, I.: RDF Support in the Virtuoso DBMS. In: Procs. of 1st Conf. on Social Semantic Web (2007)

4. Harth, A.: VisiNav: Visual web data search and navigation. In: Bhowmick, S.S., Küng, J., Wagner, R. (eds.) DEXA 2009. LNCS, vol. 5690, pp. 214-228. Springer, Heidelberg (2009)

5. Hartig, O., Bizer, C., Freytag, J.-C.: Executing SPARQL Queries over the Web of Linked Data. In: Bernstein, A., Karger, D.R., Heath, T., Feigenbaum, L., Maynard, D., Motta, E., Thirunarayan, K. (eds.) ISWC 2009. LNCS, vol. 5823, pp. 293-309. Springer, Heidelberg (2009)

6. Holi, M., Hyvönen, E.: Fuzzy view-based semantic search. In: Mizoguchi, R., Shi, Z.-Z., Giunchiglia, F. (eds.) ASWC 2006. LNCS, vol. 4185, pp. 351-365. Springer, Heidelberg (2006)

7. Kohlschütter, C.: Using link analysis to identify aspects in faceted web search. In: SIGIR 2006 Workshop on Faceted Search, pp. 55-59 (2006)

8. Lu, J., Zhu, Y., Zeng, X., Koehl, L., Ma, J., Zhang, G.: A fuzzy decision support system for garment new product development. In: Australasian Conf. on Artificial Intelligence, pp. 532-543 (2008)

${ }^{6}$ More in the extended version of this paper (on preparation). 
9. Mäkelä, E., Hyvönen, E., Saarela, S.: Ontogator - A Semantic View-Based Search Engine Service for Web Applications. In: Cruz, I., Decker, S., Allemang, D., Preist, C., Schwabe, D., Mika, P., Uschold, M., Aroyo, L.M. (eds.) ISWC 2006. LNCS, vol. 4273, pp. 847-860. Springer, Heidelberg (2006)

10. Mazzieri, M.: A Fuzzy RDF Semantics to Represent Trust Metadata. In: 1st Workshop on Semantic Web Applications and Perspectives, SWAP 2004 (2004)

11. Oren, E., Delbru, R., Decker, S.: Extending Faceted Navigation for RDF Data. In: Cruz, I., Decker, S., Allemang, D., Preist, C., Schwabe, D., Mika, P., Uschold, M., Aroyo, L.M. (eds.) ISWC 2006. LNCS, vol. 4273, pp. 559-572. Springer, Heidelberg (2006)

12. Papadakos, P., Kopidaki, S., Armenatzoglou, N., Tzitzikas, Y.: On exploiting static and dynamically mined metadata for exploratory web searching. Knowledge and Information Systems (accepted for publication in 2011) issn 0219-1377

13. Sacco, G.M.: e-RARE: Interactive Diagnostic Assistance for Rare Diseases through Dynamic Taxonomies. In: DEXA Workshops (2008)

14. Stefaner, M., Urban, T., Seefelder, M.: Elastic lists for facet browsing and resource analysis in the enterprise. In: Bhowmick, S.S., Küng, J., Wagner, R. (eds.) DEXA 2008. LNCS, vol. 5181, pp. 397-401. Springer, Heidelberg (2008)

15. Straccia, U., Lopes, N., Lukacsy, G., Polleres, A.: A general framework for representing and reasoning with annotated semantic web data. In: Twenty-Fourth AAAI Conf. on Artificial Intelligence, AAAI 2010 (2010)

16. Straccia, U.: A Minimal Deductive System for General Fuzzy RDF. In: Polleres, A., Swift, T. (eds.) RR 2009. LNCS, vol. 5837, pp. 166-181. Springer, Heidelberg (2009)

17. Tilsner, M., Hoeber, O., Fiech, A.: Cubansea: Cluster-based visualization of search results. In: Procs of the Inter. Joint Conf. on Web Intelligence and Intelligent Agent Technology, WI-IAT 2009 (2009)

18. Udrea, O., Recupero, D.R., Subrahmanian, V.S.: Annotated RDF. ACM Trans. Comput. Logic 11(2) (2010)

19. Wu, J.-K., Desai Narasimhalu, A., Mehtre, B.M., Lam, C.-P., Gao, Y.J.: Core: A content-based retrieval engine for multimedia information systems. Multimedia Syst. 3(1), 25-41 (1995)

20. Yee, K.-P., Swearingen, K., Li, K., Hearst, M.: Faceted metadata for image search and browsing. In: Procs. of the SIGCHI Conf. on Human factors in Computing Systems (2003) 